Communications in Physics, Vol. 20, No. 1 (2010), pp. 9-14

\title{
LANCZOS GENERATOR IN GÖDEL GEOMETRY
}

\author{
Z. AHSAN \\ Department of Mathematics, Aligarh Muslim University \\ Aligarh 202 002, India \\ J. H. CALTENCO, R. LINARES Y M, J. LÓPEZ-BONILLA \\ SEPI-ESIME-Zacatenco, Instituto Politécnico Nacional \\ Edif. Z-4, 3er. Piso, Col. Lindavista, CP 07738, México D.F.
}

\begin{abstract}
We exhibit three ways to obtain a Lanczos spintensor for the Gödel cosmological model. In particular, a symmetric tensor of rank two is constructed which satisfies the wave equation and generates a Lanczos potential.
\end{abstract}

\section{INTRODUCTION}

Lanczos [1-6] showed, for any $R_{4}$, the existence of a potential $K_{a b c}$ with the properties:

$$
K_{a b c}=-K_{b a c}, \quad K_{a b c}+K_{b c a}+K_{c a b}=0,
$$

which generates the conformal tensor via the expression $[2,7]$ :

$$
\begin{gathered}
C_{a b c d}=K_{a b c ; d}-K_{a b d ; c}+K_{c d a ; b}-K_{c d b ; a}+ \\
\frac{1}{2}\left[g_{a d}\left(K_{b c}+K_{c b}\right)-g_{a c}\left(K_{b d}+K_{d b}\right)+g_{b c}\left(K_{a d}+K_{d a}\right)-g_{b d}\left(K_{a c}+K_{c a}\right)\right]+ \\
\frac{2}{3}\left(g_{a c} g_{b d}-g_{a d} g_{b c}\right) K_{p ; q}^{p q},
\end{gathered}
$$

such that

$$
K_{b c} \equiv K_{b}{ }^{p}{ }_{c ; p}-K_{b}{ }^{p}{ }_{p ; c}
$$

The possible relevance of $K_{a b c}$ in general relativity is given in [8]. Besides, the idea of "Lanczos spintensor" is very useful in the analysis [9-19] of the Liénard-Wiechert field $[20]$.

Given the Weyl tensor, it may be very difficult to obtain a Lanczos superpotential by integrating directly (2), but here we shall show three ways to deduce one solution of (2) for Gödel spacetime $[21,22]$, with the interesting structure:

$$
K_{a b c}=Q_{c a ; b}-Q_{c b ; a}, \quad Q_{a b}=Q_{b a}
$$

which matches with conditions (1); therefore the symmetric tensor $Q_{a b}$ is a potential for the Lanczos generator. A relation similar to (4) also appears for weak gravitational fields [1], Kerr geometry [22-27], plane gravitational waves [28] and Kinnersley metrics [29]. The physical meaning of the Lanczos spintensor for a given spacetime is yet unknown 
$[1,6-8,24,30-32]$

\section{METHOD OF LOCAL AND ISOMETRIC EMBEDDING}

Here we show that the Gauss equation employed in the embedding of $R_{4}$ into $E_{5}$, permits to obtain a symmetric tensor $b_{i j}$ which generates a Lanczos potential for the Gödel cosmological model.

A spacetime can be embedded into $E_{5}$ if and only if there exist the second fundamental form $b_{a c}=b_{c a}$ fulfilling the Gauss-Codazzi equations [22]:

$$
\begin{gathered}
R_{a c i j}=\varepsilon\left(b_{a i} b_{c j}-b_{a j} b_{c i}\right), \\
B_{c i j} \equiv b_{j i ; c}-b_{j c ; i}=0,
\end{gathered}
$$

where $\varepsilon= \pm 1, R_{a c i j}$ is the curvature tensor and $; j$ denotes the covariant derivative. Then we say that such 4 -space has class one.

From the Gauss relation (5) it is possible to deduce the identity [33-37]:

$$
p b_{i j}=\frac{K_{2}}{48} g_{i j}-\frac{1}{2} R_{i a c j} G^{a c},
$$

where $G_{a c}=R_{a c}-\frac{R}{2} g_{a c}$ and $R_{a c}=R_{a c r}^{r}$ are the Einstein and Ricci tensors, respectively, and $K_{2}={ }^{*} R^{* i j a c} R_{i j a c}$ is a Lanczos invariant [5,38] in terms of the double dual [1] of Riemann tensor ${ }^{*} R^{* i j}{ }_{a c}=\frac{1}{4} \eta^{i j r m} R_{r m}{ }^{n r} \eta_{n r a c}$, where $\eta_{i j a c}$ is the Levi-Civita tensor. Besides:

$$
p^{2}=-\frac{\varepsilon}{6}\left(\frac{R}{24} K_{2}+R_{i m n j} G^{i j} G^{m n}\right) \geq 0,
$$

If $p \neq 0$ then (7) permits to obtain explicitly a $b_{i j}$ verifying (5).

Now we apply (7) to the Gödel metric $[21,22]$ (signature +2$)$ :

$$
d s^{2}=-\left(d x^{1}\right)^{2}-2 e^{x^{4}} d x^{1} d x^{2}-\frac{1}{2} e^{2 x^{4}}\left(d x^{2}\right)^{2}+\left(d x^{3}\right)^{2}+\left(d x^{4}\right)^{2},
$$

therefore $\varepsilon=1, p=\frac{\sqrt{2}}{4}$ and:

$$
\left(b_{i j}\right)=-\frac{\sqrt{2}}{2}\left(\begin{array}{llll}
1 & e^{x^{4}} & 0 & 0 \\
e^{x^{4}} & \frac{3}{2} e^{2 x^{4}} & 0 & 0 \\
0 & 0 & 0 & 0 \\
0 & 0 & 0 & 1
\end{array}\right) .
$$

The tensor (10) not satisfies (6) because we know [39-43] that (9) has not class one, in fact, $b_{12 ; 4} \neq b_{14 ; 2}$. Thus for this Gödel solution we have a $B_{c i j}$ whose only non-zero independent components are:

$$
B_{124}=B_{412}=-\frac{\sqrt{2}}{2} e^{x^{4}}, \quad B_{242}=\frac{3}{2} \sqrt{2} e^{2 x^{4}},
$$

with the same symmetries as Lanczos potential $K_{c i j}[6,44,45]$ :

$$
\begin{gathered}
B_{i j r}=-B_{j i r}, \quad B_{i j r}+B_{j r i}+B_{r i j}=0, \\
B_{i}{ }^{r}{ }_{r}=0 \quad \text { Lanczos algebraic gauge, }
\end{gathered}
$$




$$
B_{i j}{ }^{r} ; r=0 \quad \text { Lanczos differential gauge. }
$$

Then the following "ansatz" is very natural:

$$
K_{i j r}=Q B_{i j r}, \quad \mathrm{Q}=\text { constant, }
$$

which must generate the Weyl tensor via the relation (2) with the simplification $[24,46]$ :

$$
C_{a i j r}=K_{a i j ; r}-K_{a i r ; j}+K_{j r a ; i}-K_{j r i ; a}+g_{a r} K_{j i}-g_{a j} K_{r i}+g_{i j} K_{r a}-g_{i r} K_{j a},
$$

where $K_{i j}=K_{i}{ }_{j ; r}^{r}=K_{j i}$, because $K_{i}{ }_{r}^{r}=0$. Using (11) and (13) we find that (14) implies correctly all components of the conformal tensor if $Q=\frac{\sqrt{2}}{18}$, which means that (10) produces a Lanczos potential for Gödel geometry:

$$
K_{i j r}=\frac{\sqrt{2}}{18}\left(b_{r j ; i}-b_{r i ; j}\right)
$$

equivalent to (4) with $Q_{a c}=-\frac{\sqrt{2}}{18} b_{a c}$.

We know that (9) not accepts embedding into $E_{5}$, however, the study of the GaussCodazzi equations is important because it permits to construct the Lanczos generator (15) for the Gödel spacetime. Then, if a metric has not class one, perhaps a $b_{i j}$ verifying (5) may have a relationship similar to (15) with a Lanczos potential for this metric. Our work calls the attention towards an interesting connection between the embedding of Riemann 4 -spaces and the Lanczos generator, which must be studied carefully. For example, it is still ignored if (9) admits embedding into $E_{6}$ [41-43,47], then the Lanczos potential gives us a new approach to this open problem.

\section{METHOD OF THE LOVELOCK'S THEOREM}

The Lanczos algebraic gauge $K_{a}{ }^{b}{ }_{b}=0$ may be satisfied if in (4) we ask the conditions:

$$
\begin{gathered}
Q_{r}^{r}=\text { constant, } \\
Q_{a}{ }^{b} ; b=0 ; .
\end{gathered}
$$

Besides, if now we suppose that $Q_{a b}$ depends locally on the intrinsic geometry of $R_{4}$, that is:

$$
Q_{i r}=Q_{i r}\left(g_{a b} ; g_{a b, c} ; g_{a b, c d}\right)
$$

then a Lovelock's theorem [34,48-50] affirms that in four dimensions a tensor of second rank with the properties (17) and (18) must have the form:

$$
Q_{a b}=\alpha G_{a b}+\beta g_{a b}, \quad \alpha, \beta=\quad \text { constant }
$$

Thus $Q_{r}{ }^{r}=4 \beta-\alpha R$ satisfies (16) because $R=1$ for the Gödel metric (9); if we put (19) into (4) it results:

$$
K_{a b c}=\alpha\left(R_{c a ; b}-R_{c b ; a}\right)
$$


Finally with the help of (9), (14) and (20) we conclude that $\alpha=-\frac{1}{9}$, that is [51-53]:

$$
K_{i j r}=\frac{1}{9}\left(R_{r j ; i}-R_{r i ; j}\right)
$$

which is the same as (15) because $b_{a c}$ is connected with $R_{a c}$ by the expression:

$$
b_{i j}=\sqrt{2}\left[R_{i j}+\frac{1}{2}\left(B_{i} B_{j}-g_{i j}\right)\right],
$$

where $B_{j}$ is a constant spacelike vector:

$$
\left(B_{r}\right)=(0,0,1,0), \quad B_{r ; c}=0 .
$$

It is interesting to observe that $K_{a b c} v^{c} \propto \omega_{a b}$, where $\left(v^{c}\right)=(1,0,0,0)$ is the velocity of the fluid and $\omega_{i j}$ is the spin [54] of the matter rotating in this Gödel model, in accordance with the proposition [10] of that $K_{i j r}$ represents some type of angular momentum into the spacetime.

\section{METHOD OF THE WAVE EQUATION}

Here we consider symmetric tensors of second order verifying the wave equation:

$$
W_{a b}{ }_{; r}=0,
$$

in the Gödel spacetime, and we exhibit an attractive solution of (24) which generates a Lanczos potential for (9) with the structure (4).

In fact, (24) admits the solution:

$$
\left(W_{a b}\right)=\frac{1}{27}\left(\begin{array}{llll}
4 & 4 e^{x^{4}} & 0 & 0 \\
4 e^{x^{4}} & \frac{7}{2} e^{2 x^{4}} & 0 & 0 \\
0 & 0 & 0 & 0 \\
0 & 0 & 0 & -1
\end{array}\right), \quad W_{a}{ }^{b}{ }_{; b}=0
$$

then

$$
K_{i j r}=W_{r i ; j}-W_{r j ; i}
$$

implies a Lanczos generator whose only non-zero independent components are:

$$
K_{124}=K_{412}=-\frac{e^{x^{4}}}{18}, \quad K_{242}=\frac{e^{2 x^{4}}}{6},
$$

with all properties (12), which reproduces -via (14)- correctly the Weyl tensor. The Lanczos potential (26) is equivalent to (15) due the connection:

$$
W_{r i}=\frac{1}{18}\left[-\sqrt{2} b_{r i}+\frac{5}{3}\left(B_{r} B_{i}-g_{r i}\right)\right] .
$$

Our work shows that it is important to study the wave equation (24) for the construction of $K_{i j r}$ in Gödel geometry, but it is clear that this situation may appear in other spacetimes. 


\section{REFERENCES}

[1] Lanczos, C., Rev. Mod. Phys. 34 (1962) 379-389.

[2] Bampi, F. and Caviglia, G., Gen. Rel. Grav. 15 (1983) 375-386.

[3] Illge, R., Gen. Rel. Grav. 20 (1988) 551-564.

[4] López-Bonilla, J., Morales, J., Navarrete, D. and Rosales, M., Class. Quant. Grav. 10 (1993) 21532155.

[5] Gaftoi, V., López-Bonilla, J. and Ovando, G., Nuovo Cim. B113 (1998) 1489-1492.

[6] Gaftoi, V., López-Bonilla, J. and Ovando, G., Nuovo Cim. B113 (1998) 1493-1496.

[7] Roberts, M. D., Gen. Rel. Grav. 20 (1988) 775-792.

[8] Gaftoi, V., López-Bonilla, J., Morales, J., Ovando, G. and Peña, J. J., J. Moscow Phys. Soc. 6 (1996) 267-278.

[9] Aquino, N., López-Bonilla, J., Núñez-Yépez, H. N. and Salas-Brito, A. L., J. Phys. A: Math. Gen. 28 (1995) L375-L379.

[10] López-Bonilla, J., Ovando, G. and Rivera, J., Nuovo Cim. B112 (1997) 1433-1436.

[11] López-Bonilla, J., Ovando, G. and Rivera, J., Indian J. Pure Appl. Math. 28 (1997) 1355-1360.

[12] López-Bonilla, J., Núñez-Yépez, H. N. and Salas-Brito, A. L., J. Phys. A: Math. Gen. 30 (1997) 3663-3669.

[13] Gaftoi, V., López-Bonilla, J. and Ovando, G., Int. J. Theor. Phys. 38 (1999) 939-943.

[14] López-Bonilla, J. and Ovando, G., Gen. Rel. Grav. 31 (1999) 1931-1934.

[15] Arreaga, G., López-Bonilla, J. and Ovando, G., Indian J. Pure Appl. Math. 31 (2000) 85-91.

[16] López-Bonilla, J., Morales, J. and Ovando, G., Indian J. Phys. B74 (2000) 167-169.

[17] Caltenco, J. H., López-Bonilla, J. and Peña-Rivero, R., Indian J. Theor. Phys. 50 (2002) 1-3.

[18] Caltenco, J. H., López-Bonilla, J., Morales, J. and Ovando, G., Chinese J. Phys. 40 (2002) 214-222.

[19] Acevedo, M., López-Bonilla, J. and Sosa-Pedroza, J., Apeiron 9 (2002) 43-48.

[20] López-Bonilla, J., Morales, J. and Rosales, M., Pramana J. Phys. 42 ( 1994) 89-95.

[21] Gödel, K., Rev. Mod. Phys. 21 (1949) 447-450.

[22] Kramer, D., Stephani, H., MacCallum, M. and Herlt, E., Exact solutions of Einstein's field equations, Cambridge Univ. Press, Cambridge, 1980.

[23] Kerr, R. P., Phys. Rev. Lett. 11 (1963) 237-238.

[24] López-Bonilla, J., Morales, J. and Ovando, G., Gen. Rel. Grav. 31 (1999) 413-415.

[25] Caltenco, J. H., López-Bonilla, J., Morales, J. and Ovando, G., Aligarh Bull. Math. 19 (2000) $49-54$.

[26] Caltenco, J. H., López-Bonilla, J., Morales, J. and Ovando, G., Chinese J. Phys. 39 (2001) 397-400.

[27] Acevedo, M., López-Bonilla, J. and Vidal-Beltrán, S., Grav. 6 Cosm. 10 (2004) 328-328

[28] López-Bonilla, J., Ovando, G. and Peña, J. J., Found. Phys. Lett. 12 (1999) 401-405.

[29] Caltenco, J. H., López-Bonilla, J., Ovando. G. and Rivera, J., Apeiron 9 (2002) 38-42.

[30] De Freitas, L. R., Novello, M. and Pinto-Neto, N., J. Math. Phys. 35 (1994) 734-747.

[31] Roberts, M. D., Nuovo Cim. B110 (1995) 1165-1176.

[32] Bergqvist, G., J. Math. Phys. 38 ( 1997) 3142-3154.

[33] Goenner, H. F., Tensor N. S. 30 (1976) 15.

[34] Fuentes, R., López-Bonilla, J., Ovando, G. and Matos, T., Gen. Rel. Grav. 21 (1989) 777-784.

[35] González, G., López-Bonilla, J. and Rosales, M., Pramana J. Phys. 42 (1994) 85-88.

[36] López-Bonilla, J. and Núñez-Yépez, H. N., Pramana J. Phys. 46 (1996) 219-221.

[37] López-Bonilla, J., Morales, J. and Ovando, G., Indian J. Math. 42 (2000) 309-312.

[38] Lanczos, C., Ann. Math. 39 (1938) 842-850.

[39] Szekeres, P., Nuovo Cim. A43 (1966) 1062.

[40] Eguchi, K., J. Math. Tokushima Univ. 1 (1967) 17-27.

[41] Collinson, C. D., J. Math. Phys. 9 (1968) 403-410.

[42] López-Bonilla, J., Morales, J. and Rosales, M. Braz. J. Phys. 24 (1994) 522-525.

[43] López-Bonilla, J., Ovando, G. and Rivera, J., Aligarh Bull. Math. 17 (1997-98) 63-66.

[44] Edgar, S. B. and Höglund, A., Proc. R. Soc. Lond. A 453 (1997) 835-851.

[45] O’Donell, P. J., Gen. Rel. Grav. 36 (2004) 1415-1422. 
[46] O’Donell, P. J., Czech. J. Phys. 54 (2004) 889-896.

[47] Rosen, J., Rev. Mod. Phys. 37 (1965) 204-214.

[48] Lovelock, D., J. Math. Phys. 13 (1972) 874-876.

[49] Gaftoi, V., López-Bonilla, J. and Ovando, G., Bull. Allahabad Math. Soc. 14 (1999) 41-44.

[50] López-Bonilla, J., Morales, J. and Ovando, G., Indian J. Phys. B74 (2000) 397-398.

[51] López-Bonilla, J. and Ovando, G., Gen. Rel. Grav. 31 (1999) 1071-1074.

[52] López-Bonilla, J., Morales, J., Ovando, G. and Rivera-Rebolledo, J., Indian J. Theor. Phys. 48 (2000) 289-298.

[53] Gaftoi, V., López-Bonilla, J. and Ovando, G., Czech. J. Phys. 52 (2002) 811-813.

[54] Raychaudhuri, A. Relativistic Cosmology. Phys. Rev. 98 (1955) 1123-1126.

Received 15 September 2008. 